\title{
An in silico MS/MS library for automatic annotation of novel FAHFA lipids
}

\author{
Yan Ma' , Tobias Kind ${ }^{1}$, Arpana Vaniya ${ }^{1}$, Ingrid Gennity ${ }^{1}$, Johannes F. Fahrmann ${ }^{1}$ and Oliver Fiehn ${ }^{1,2^{*}}$
}

\begin{abstract}
Background: A new lipid class named 'fatty acid esters of hydroxyl fatty acids' (FAHFA) was recently discovered in mammalian adipose tissue and in blood plasma and some FAHFAs were found to be associated with type 2 diabetes. To facilitate the automatic annotation of FAHFAs in biological specimens, a tandem mass spectra (MS/MS) library is needed. Due to the limitation of the commercial available standard compounds, we proposed building an in silico MS/MS library to extend the coverage of molecules.
\end{abstract}

Results: We developed a computer-generated library with 3267 tandem mass spectra (MS/MS) for 1089 FAHFA species. FAHFA spectra were generated based on authentic standards with negative mode electrospray ionization and 10, 20 , and $40 \mathrm{~V}$ collision induced dissociation at 4 spectra/s as used in in ultra-high performance liquid chromatographyQTOF mass spectrometry studies. However, positional information of the hydroxyl group is only obtained either at lower QTOF spectra acquisition rates of 1 spectrum/s or at the $\mathrm{MS}^{3}$ level in ion trap instruments. Therefore, an additional set of 4290 fragment-rich MS/MS spectra was created to enable distinguishing positional FAHFA isomers. The library was generated based on ion fragmentations and ion intensities of FAHFA external reference standards, developing a heuristic model for fragmentation rules and extending these rules to large swaths of computer-generated structures of FAHFAs with varying chain lengths, degrees of unsaturation and hydroxyl group positions. Subsequently, we validated the new in silico library by discovering several new FAHFA species in egg yolk, showing that this library enables high-throughput screening of FAHFA lipids in various biological matrices.

Conclusions: The developed library and templates are freely available for commercial or noncommercial use at http://fiehnlab.ucdavis.edu/staff/yanma/fahfa-lipid-library. This in silico MS/MS library allows users to annotate FAHFAs from accurate mass tandem mass spectra in an easy and fast manner with NIST MS Search or PepSearch software. The developing template is provided for advanced users to modify the parameters and export customized libraries according to their instrument features.

Keywords: In silico library, MS/MS, Lipids, FAHFA

\section{Background}

Recently, a novel lipid class named 'fatty acid esters of hydroxyl fatty acids' (FAHFA) was discovered in mice adipose tissues [1]. Specifically, a FAHFA comprised of palmitic acid (16:0) esterified to a 9-hydroxyl stearic acid (9-O-18:0), abbreviated as 9-PAHSA, was discussed as promoting anti-diabetic and anti-inflammatory effects [1]. PAHSA levels were shown to be highly correlated

\footnotetext{
*Correspondence: ofiehn@ucdavis.edu

${ }^{2}$ Department of Biochemistry, Faculty of Sciences, King Abdulaziz

University, Jeddah, Saudi Arabia

Full list of author information is available at the end of the article
}

with insulin sensitivity in humans [1]. However, it remained unclear if other FAHFAs might exert similar effects, and how many different FAHFAs in total might be present in mammalian tissues or biofluids.

Currently only 21 external reference standards are commercially available. In order to enable extensive profiling and automatic annotation of FAHFA species, an MS/MS library with more structural diversity is needed. Today, such mass spectral libraries can be created by applying rules of fragmentation patterns on large in silico structure list, as we have previously shown for over 200,000 mass spectra in LipidBlast [2] for twenty-six common lipid classes such as (lyso) phosphatidylcholines,
Chemistry Central creativecommons.org/licenses/by/4.0/), which permits unrestricted use, distribution, and reproduction in any medium, provided you give appropriate credit to the original author(s) and the source, provide a link to the Creative Commons license, and indicate if changes were made. The Creative Commons Public Domain Dedication waiver (http://creativecommons.org/publicdomain/ zero/1.0/) applies to the data made available in this article, unless otherwise stated. 
monogalactosyldiacylglycerols or triacylglycerols. LipidBlast itself has been applied for the annotation of lipids in mouse liver [3], rat urine/serum [4] and in various algae species $[5,6]$, demonstrating that this strategy enables rapid annotation of many molecular species from mass spectra [7]. LipidBlast templates use heuristic information of MS/MS fragmentation patterns to extend the range of in silico predicted mass spectra that can be used to discover species of novel lipid classes. An example has been shown for glucuronosyldiacylglycerol lipids in plants [8]. Here we used the modified LipidBlast templates to build an in silico MS/MS library for 1089 species of the novel FAHFA lipid class and demonstrate the applicability of this new library.

\section{Results and discussion}

Negative mode electrospray in silico MS/MS spectra were modelled based on the reference spectra of 9-PAHSA under 10, 20, and $40 \mathrm{~V}$ collision induced dissociation (CID) voltages acquired with UHPLC-QTOF MS/MS profiling methods at 4 spectra/s (Fig. 1a; Additional file 1). Under these conditions, major FAHFA fragment ions include the precursor ion, the fatty acid fragment ion, and the hydroxyl fatty acid fragment ion including its dehydration product. No major differences in fragmentations were observed for either collision voltage, except for decreasing intensity of the precursor ion. Several isotopic peaks were observed for both precursor and fragment ions due to the insufficient isolation prior to the collision induced dissociation; however, such isotope ions were excluded from in silico fragmentation modeling since they could have been avoided with a narrower isolation width. Acquired 9-PAHSA spectra were compared to the published 9-PAHSA MS/MS spectrum and corresponding multiple reaction monitoring (MRM) fragmentation transitions [1]. While the major fragmentation ions observed in our laboratory were consistent with the published spectra, importantly, the positional fragments of the hydroxyl 18:0 fatty acid $(\mathrm{m} / z 127$ and 155$)$ were not observed. To generate those fragments (Fig. 1b), MS/MS experiments with a longer acquisition time of 1 spectrum/s were performed at $40 \mathrm{~V}$ CID, and $\mathrm{m} / z 127.133$ and 155.144 were observed at relative abundance of $0.2 \%$. The intensity of such secondary fragmentation products could not be enhanced by increasing collision voltages on the MS/MS level with QTOF MS/MS acquisition time of 4 spectra/s. Indeed, these low abundant fragments were proven to be secondary fragmentation products of MS/ MS ions by using $\mathrm{MS}^{3}$ fragmentation of the corresponding MS/MS ions, using direct infusion of the standards into a ThermoScientific Linear Ion Trap LTQ mass spectrometer (Fig. 1c).

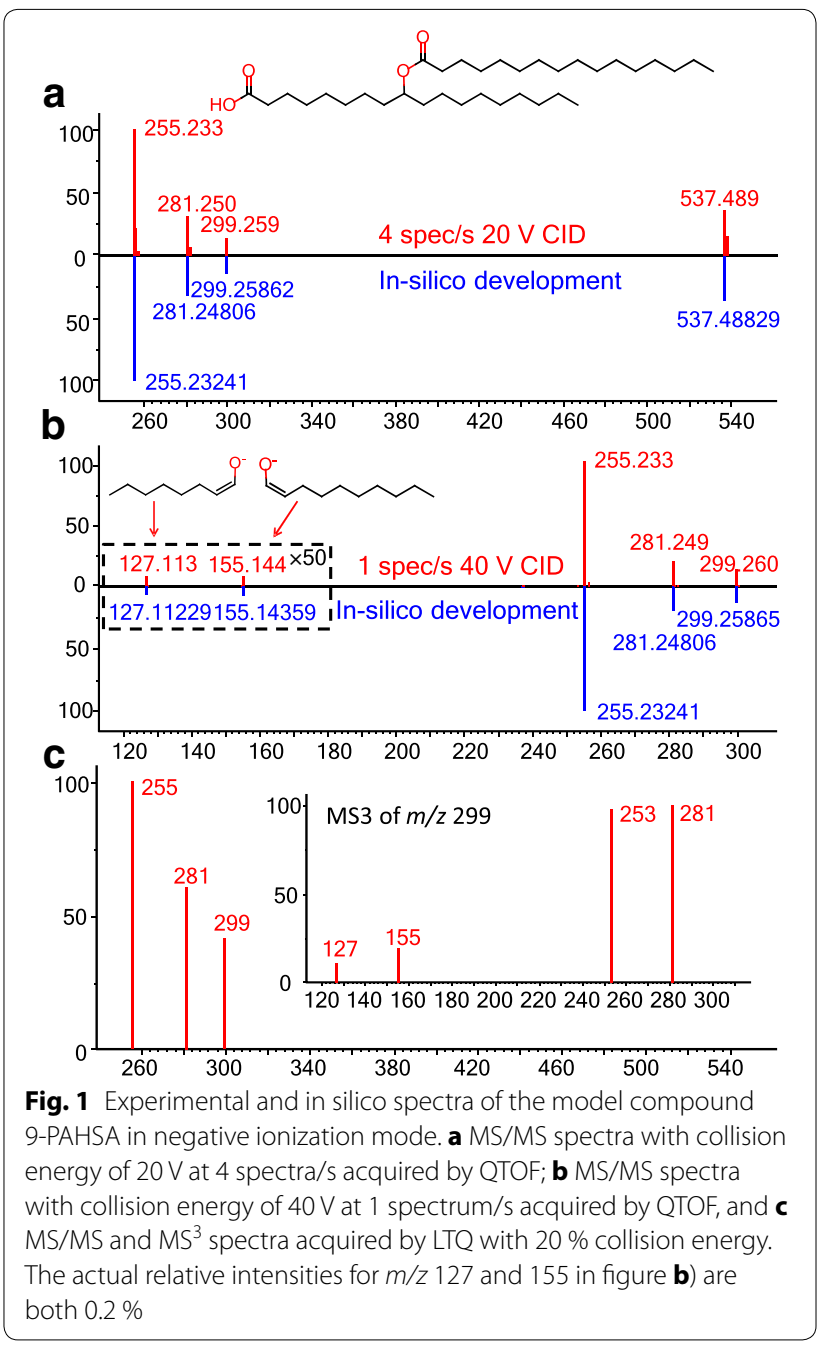

To expand the overall structure space of the FAHFA structures that can be annotated by mass spectrometry, we used 33 fatty acids commonly found in mammalian cells, varying from 14:0-24:6 (Additional file 2) for both the free fatty acid and the hydroxyl fatty acid moieties [9-11]. 1089 general FAHFA structures were defined and 3267 in silico spectra were modelled based on the fragmentation pattern of 9-PAHSA observed at three collision energies. Since the positional information of double-bonds and hydroxyl groups could not be reflected by the reference spectra acquired with the fast 4 spectra/s QTOF MS/MS profiling method, such detailed information was not specified in the structures of FAHFAs. Therefore, the structures in this FAHFA profiling library are general, semi-characterized structures. To characterize the position of the hydroxyl group, we built a more specific in silico library based on the fragment-rich spectra at $40 \mathrm{~V}$ modeled from 1 spectra/s QTOF MS/MS acquisition time. According to a patent on FAHFA lipids 
[12], the hydroxyl group may be positioned on all carbons except for the terminal carbon. Correspondingly, 4290 structures with saturated hydroxyl fatty acids and saturated or unsaturated fatty acid esters were defined, and their in silico spectra were created based on the reference spectra of 9-PAHSA acquired with 1 spectrum/s MS/ MS method. Due to a lack of published spectra or commercially available standards, modeling for unsaturated hydroxyl fatty acid residues was excluded. To further verify the position of the hydroxyl group we used relative retention time information. For the elution order of three commercially available OAHSA isomer reference standards, we observed increasing retention times when the hydroxyl group was positioned closer to the carboxylic acid moiety, with 12-OAHSA eluting at $6.07 \mathrm{~min}$, 9-OAHSA at $6.21 \mathrm{~min}$ and 5-OAHSA eluting at $6.45 \mathrm{~min}$ under the conditions described in the experimental section. For building a reliable retention time database or even predicting retention times for other FAHFAs, a larger range of authentic reference standards would be needed.

The experimental MS/MS spectra of 5-OAHSA, 9-OAHSA and 12-OAHSA (Additional file 1) were used for validation of the in silico library as they had not been employed in the library generation. Using NIST MS Search, all general structures were correctly annotated by the in silico FAHFA library with a Reverse-Dot score of greater than 950. Figure 2 shows an example of the annotation of 12-OAHSA at 20 and $40 \mathrm{~V}$ using 4 and 1 spectra/s acquisition rates. For each case, the correct FAHFA was identified, and when using the longer MS/MS acquisition time, the correct isomer was identified, even when there were slight differences between predicted and experimentally observed ion intensities at specific collision induced voltages. As further validation, negative mode MS/MS spectra of eight published FAHFAs from the METLIN [13] database were also successfully annotated using the in silico library (Additional file 3).
To validate the usefulness of this new in silico FAHFA library, we analyzed complex lipids extracted from egg yolk and annotated FAHFAs by matching experimental to predicted MS/MS spectra. PAHSA isomer levels in egg yolk were measured using multiple reaction monitoring (MRM) in the previously published report [1], but no other FAHFA family members were reported in egg yolk. With the in silico library, we successfully annotated six abundant FAHFAs in egg yolk with $<5 \mathrm{mDa}$ errors for the precursor ions and Reverse-Dot scores greater than 900 for the MS/MS matching, including four FAHFA lipids that have never been detected before (Table 1; Fig. 3). As example for such novel FAHFA lipids, Fig. 4 compares the experimental to the in silico MS/MS spectra of FAHFA 18:2-(O-18:1), or LAHOA, at a scan rate of 4 spectra/s and $20 \mathrm{~V}$ collision energy. Here the positional fragments were not detected, suggesting that it is very challenging to obtain the positional information from complex mixtures in fast-scanning LC-MS/MS experiments. These examples demonstrate that in silico libraries such as the FAHFA library created here are suitable to annotate novel compounds detected in untargeted UHPLC-QTOF MS/MS profiling studies. We suggest that this library may be used in studies investigating the biological functions, regulation and distribution of FAHFAs.

\section{Conclusions}

We developed an in silico MS/MS library for FAHFA lipids with a total of 7557 QTOF spectra in negative ionization mode. The new library enables users to automatically annotate FAHFAs in LC-MS/MS lipidomics profiling and can be therefore applied to further studies of this novel lipid class. The batch annotation process is very easy and fast using NIST MS PepSearch. We also provided the Excel template for users to adapt this library to their own instrument features and parameters and export customized libraries. The developed library and templates are freely available for commercial or
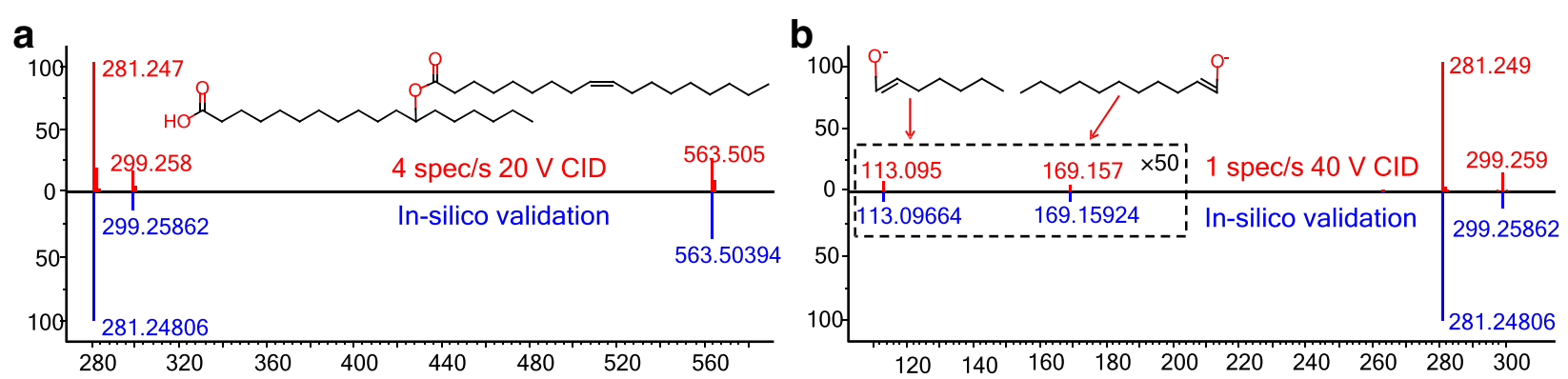

Fig. 2 Experimental and in silico MS/MS spectra of one standard for validation 12-OAHSA in negative ionization mode. Spectra were acquired at a collision energy of $20 \mathrm{~V}$ at 4 spectra/s and $\mathbf{b}$ collision energy of $40 \mathrm{~V}$ at 1 spectrum/s. The actual relative intensities for $\mathrm{m} / \mathrm{z} 113 \mathrm{and} 169$ are 0.2 and $0.1 \%$, respectively 
Table 1 Information about FAHFAs found in egg yolk

\begin{tabular}{lllll}
\hline Name & Abbrev. & RT $(\mathbf{m i n})$ & $\mathbf{m} / \mathbf{z}$ & Rev-dot \\
\hline 16:0-(O-18:1) & PAHOA & 5.61 & 535.471 & 910 \\
18:1-(O-18:1) & OAHOA & 5.61 & 561.485 & 987 \\
18:1-(O-18:2) & OAHLA & 5.42 & 559.469 & 994 \\
18:2-(O-18:1) & LAHOA & 5.08 & 559.469 & 999 \\
18:2-(O-18:2) & LAHLA & 4.87 & 557.454 & 997 \\
18:3-(O-18:1) & ALAHOA & 4.67 & 557.454 & 957 \\
\hline
\end{tabular}

$R T$ retention time

$\mathrm{m} / \mathrm{z}$ can be used for annotation in future LC-MS profiling experiments

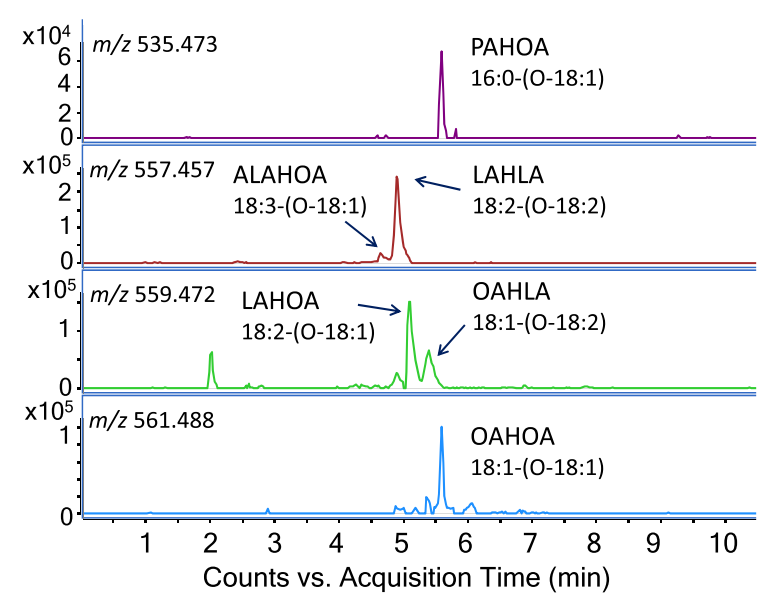

Fig. 3 Extracted ion chromatograms (EICs) for FAHFAs found in negative ionization mode in egg yolk. The $\mathrm{m} / \mathrm{z}$ tolerance was 0.005 Da

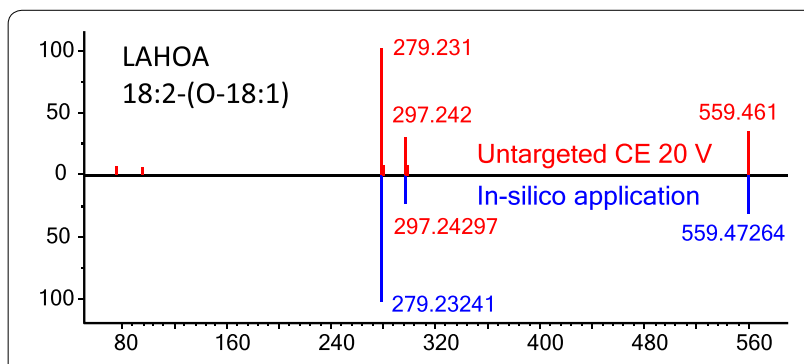

Fig. 4 MS/MS spectra of FAHFA 18:2-(O-18:1) in negative ionization mode, annotated in the egg yolk by 4 spectra/s MS/MS method. Position of hydroxyl group is not specified. The experimental spectra have two extra peaks m/z 75.751 and 95.822, which may come from the background noise or other co-eluting compounds

noncommercial use under creative commons-by attribution (CC-BY) license and can be downloaded from http:// fiehnlab.ucdavis.edu/staff/yanma/fahfa-lipid-library. The subset of 4290 spectra with defined structures (provided as InChI codes) is also available in Massbank of North America (MoNA) at http://mona.fiehnlab.ucdavis.edu/.

\section{Methods}

\section{Experimental measurements of standards}

FAHFA standards, including 9-PAHSA [16:0-(9-O-18:0)], 5-OAHSA [18:1-(5-O-18:0)], 9-OAHSA [18:1-(9-O18:0)], and 12-OAHSA [18:1-(12-O-18:0)], were purchased from Cayman Chemical (Ann Arbor, MI). Stock solution for each lipid standard was prepared in ethanol at $1 \mathrm{mg} / \mathrm{mL}$, and then diluted in methanol to $50 \mathrm{ppm}$ for injection. LC-MSMS acquisition was performed by an Agilent 1290 HPLC coupled to an Agilent 6530 quadrupole time of flight (QTOF) mass spectrometer. A Waters Acquity CSH C18 column $(2.1 \times 100 \mathrm{~mm}, 1.7 \mu \mathrm{M})$ was used for separation. Mobile phase A consisted of 60:40 acetonitrile:water while mobile phase B consisted of 90:10 isopropanol:acetonitrile, both with $9.2 \mathrm{mM}$ ammonium acetate. Column temperature was set to $65^{\circ} \mathrm{C}$ and the flow rate was $0.6 \mathrm{~mL} / \mathrm{min}$. The following gradient was applied: 0-2 min from $15 \% \mathrm{~B}-30 \% \mathrm{~B}, 2-2.5 \mathrm{~min}$ from $30 \% \mathrm{~B}-48 \% \mathrm{~B}, 2.5-11 \mathrm{~min}$ from $48 \% \mathrm{~B}-82 \% \mathrm{~B}$, 11-11.5 min from 82-99\% B, 11.5-12 min remain $99 \%$ B, 12-12.1 from $99 \% \mathrm{~B}-15 \% \mathrm{~B}, 12.1-15$ min re-equilibrate at $15 \%$ B. $3 \mu \mathrm{L}$ of each standard as well as a mixture of the four standards were injected. MS and MS/MS data was collected in negative ionization mode, in profile and centroid mode with a scan rate of 4 spectra per second. Multiple collision energies were applied, including 10,20 and $40 \mathrm{~V}$. In addition, MS/MS data was acquired specifically for $m / z 537.489$ and $m / z 563.504$ at acquisition time of 1 spectrum per second. MS/MS spectra were exported from Agilent MassHunter software to Mascot Generic Format (MGF) format. Additional confirmation was performed by MS/MS and $\mathrm{MS}^{3}$ experiments by direct infusion of $10 \mathrm{ppm}$ standard solutions in methanol into a ThermoScientific Linear Ion Trap (LTQ) mass spectrometer at $5 \mathrm{kV}$ electrospray voltage and $30 \mathrm{~ms}$ collision activation time with an activation $\mathrm{Q}$ set at 0.25 , isolation width of $3 \mathrm{Da}$ and the normalized collision energy for collision induced dissociation was set to $20 \%$ for the acquisition of $\mathrm{MS}^{3}$ level spectra.

\section{In-silico development and validation}

10, 20 and $40 \mathrm{~V} \mathrm{MS/MS} \mathrm{spectra} \mathrm{of} \mathrm{9-PAHSA} \mathrm{were} \mathrm{used}$ for the development of the in silico library. Fragmentation patterns were manually investigated, and resulting $\mathrm{m} / \mathrm{z}$ and abundance of all major peaks at each collision energy were included in the modified LipidBlast template. Molecular formula and accurate masses were calculated from chain lengths and degrees of unsaturation of the fatty acid and hydroxyl fatty acid residues, using the Exact Mass Calculator [14]. To expand the structure space of the library, a series of common fatty acids found in mammalian systems were added to the template, as fatty acid moieties [9-11]. Spectra information was calculated 
according to the model compound. Fatty acid structures were downloaded from the lipid metabolites and pathways strategy (Lipid MAPS) database [15]. Hydroxyl fatty acid and FAHFA structures were generated by ChemAxon Marvin 9.5.3 and JChem Reactor 9.5.3 [16]. VBA code of LipidBlast was modified to fit the new template and export the spectra to NIST MSP format. MSP file was then converted to NIST library by Lib2NIST [17] software and was ready to be used with NIST MS Search or NIST MS PepSearch software [18]. Other internal experimental MS/MS spectra as well as the external spectra from METLIN [13] online database were used for validation.

\section{Application: lipidomics of egg yolk}

With slight modifications, lipid extraction of egg yolk was performed according to a previously published method [1]. Briefly, $300 \mu \mathrm{L}$ egg yolk was added to $1200 \mu \mathrm{L}$ citric acid buffer $(100 \mathrm{mM}$ sodium citrate, $1 \mathrm{M} \mathrm{NaCl}$ ), followed by adding $1.5 \mathrm{~mL}$ of methanol and $3 \mathrm{~mL}$ of chloroform. The mixture was shaken by hand for $30 \mathrm{~s}$, vortexed for $15 \mathrm{~s}$, and centrifuged at $2200 \mathrm{~g}, 4^{\circ} \mathrm{C}$ for $6 \mathrm{~min}$. The organic phase was dried under a gentle stream of nitrogen gas. The extracted lipids were reconstituted with $200 \mu \mathrm{L}$ chloroform and loaded to a pre-conditioned SPE cartridge (500 mg silica, $6 \mathrm{~mL}$, Thermo Scientific). Neutral lipids were eluted with $15 \mathrm{~mL} 5 \%$ ethyl acetate in hexane followed by the elution of FAHFAs with $15 \mathrm{~mL}$ ethyl acetate. The FAHFA lipid fraction was dried under nitrogen gas and stored at $-80^{\circ} \mathrm{C}$ prior to LC/MS analysis. On the day of the experiment, the lipid extract was re-suspended with $30 \mu \mathrm{L}$ of methanol and $5 \mu \mathrm{L}$ was injected. The LC-MSMS method was similar to the method for the reference standard measurements, with a scan rate of 4 spectra per second and collision energy of 20 V. NIST MS PepSearch was used for the MS/MS search against the in silico FAHFA library.

\section{Additional files}

Additional file 1. Experimental MS/MS spectra of 9-PAHSA, 5-OAHSA, 9-OAHSA, and 12-OAHSA from Cayman Chemical, acquired with 10, 20, $40 \mathrm{~V} \mathrm{CID}$ at $4 \mathrm{spectra} / \mathrm{s}$ and $40 \mathrm{~V}$ CID at 1 spectrum $/ \mathrm{s}$.

Additional file 2. Common fatty acids included in the FAHFA in silico library.

Additional file 3. Annotation results of the METLIN online reference spectra by the in silico library.

\section{Authors' contributions}

YM, TK, OF designed the experiment. YM developed and validated the in silico library. YM, AV, IG and JFF performed experimental measurements. YM, TK and OF wrote the manuscript. All authors read and approved the final manuscript.

\section{Author details}

${ }^{1}$ UC Davis Genome Center-Metabolomics, Davis, CA, USA. ${ }^{2}$ Department of Biochemistry, Faculty of Sciences, King Abdulaziz University, Jeddah, Saudi Arabia.

\section{Acknowledgements}

This study was funded by National Science Foundation grant MCB 1139644 and National Institutes of Health Grant U24 DK097154 and 1S1ORR031630.

\section{Competing interests}

The authors declare that they have no competing interests.

Received: 13 August 2015 Accepted: 3 November 2015

Published online: 16 November 2015

\section{References}

1. Yore MM, Syed I, Moraes-Vieira PM, Zhang T, Herman MA, Homan EA, Patel RT, Lee J, Chen S, Peroni OD (2014) Discovery of a class of endogenous mammalian lipids with anti-diabetic and anti-inflammatory effects. Cell 159(2):318-332

2. Kind T, Liu KH, Lee do Y, DeFelice B, Meissen JK, Fiehn O (2013) Lipidblast in silico tandem mass spectrometry database for lipid identification. Nat Methods 10(8):755-758

3. Park HM, Shon JC, Lee MY, Liu K-H, Kim JK, Lee SJ, Lee CH (2014) Mass spectrometry-based metabolite profiling in the mouse liver following exposure to ultraviolet B radiation. PLoS One 9(10):e109479

4. Kim HY, Lee MY, Park HM, Park YK, Shon JC, Liu KH, Lee CH (2015) Urine and serum metabolite profiling of rats fed a high-fat diet and the antiobesity effects of caffeine consumption. Molecules 20(2):3107-3128

5. Ogawa T, Furuhashi T, Okazawa A, Nakai R, Nakazawa M, Kind T, Fiehn O, Kanaya S, Arita M, Ohta D (2014) Exploration of polar lipid accumulation profiles in Euglena gracilis using LipidBlast, an MS/MS spectral library constructed in silico. Biosci Biotechnol Biochem 78(1):14-18

6. Kind T, Meissen JK, Yang D, Nocito F, Vaniya A, Cheng YS, VanderGheynst JS, Fiehn O (2012) Qualitative analysis of algal secretions with multiple mass spectrometric platforms. J Chromatogr A 1244:139-147

7. Tsugawa H, Cajka T, Kind T, Ma Y, Higgins B, Ikeda K, Kanazawa M, VanderGheynst J, Fiehn O, Arita M (2015) MS-DIAL: data-independent MS/MS deconvolution for comprehensive metabolome analysis. Nat Methods 12(6):523-526

8. Kind T, Okazaki Y, Saito K, Fiehn O (2014) LipidBlast templates as flexible tools for creating new in-silico tandem mass spectral libraries. Anal Chem 86(22):11024-11027

9. McEvoy T, Coull G, Broadbent P, Hutchinson J, Speake B (2000) Fatty acid composition of lipids in immature cattle, pig and sheep oocytes with intact zona pellucida. J Reprod Fertil 118(1):163-170

10. Käkelä R, Hyvärinen H (1996) Site-specific fatty acid composition in adipose tissues of several northern aquatic and terrestrial mammals. Comp Biochem Physiol B: Biochem Mol Biol 115(4):501-514

11. Svennerholm L (1968) Distribution and fatty acid composition of phosphoglycerides in normal human brain. J Lipid Res 9(5):570-579

12. Kahn B, Herman M, Saghatelian A, Homan E Lipids that increase insulin sensitivity and methods of using the same. Google Patents, 2013

13. Smith CA, O'Maille G, Want EJ, Qin C, Trauger SA, Brandon TR, Custodio DE, Abagyan R, Siuzdak G (2005) METLIN: a metabolite mass spectral database. Ther Drug Monit 27(6):747-751

14. Exact Mass Calculator. http://www.sisweb.com/referenc/tools/exactmass. htm. Accessed 27 Feb 2015

15. Fahy E, Sud M, Cotter D, Subramaniam S (2007) LIPID MAPS online tools for lipid research. Nucleic Acids Res 35(suppl 2):W606-W612

16. ChemAxon. http://www.chemaxon.com. Accessed 9 Mar 2015

17. Lib2NIST. http://chemdata.nist.gov/. Accessed 3 Apr 2015

18. Stein SE, Scott DR (1994) Optimization and testing of mass spectral library search algorithms for compound identification. J Am Soc Mass Spectrom 5(9):859-866 